\title{
Aktivierung von Biopterin in der Ratte
}

\author{
Heinz Rembold und Heinz Metzger \\ Max-Planck-Institut für Biochemie, München
}

(Z. Naturforschg. 22 b, 827-830 [1967] ; eingegangen am 25. Januar 1967)

\begin{abstract}
$8 \mathrm{a}-{ }^{14} \mathrm{C}$-Biopterin intraperitoneally injected into rats is secreted within a short time and in a nearly quantitative amount into the urine. After feeding, $90 \%$ of the substance remains in the organism. 7-biopterin is absorbed only in small amounts and is excreted to a large extent with the feces. The absorbed labeled biopterin is equally distributed throughout the body. After three days a measureable amount of activity is found in the serum as well as in the liver and adrenals. The same level of activity occurs in regenerating liver and ascites tumour cells. After chromatographic separation of a $5 \%$ TCA soluble extract obtained from $250 \mathrm{~g}$ of rat livers, only $10 \%$ of the total radioactivity was found in biopterin. The remaining activity appears mainly in a weakly basic, extremely labile component. Other pterines could not be demonstrated. No biopterin is found in the TCA insoluble liver fraction. The compound occurs as a tetrahydrobiopterin in the liver in a concentration of about $75 \mu \mathrm{g}$; biopterin exists only in trace amounts. After fractional centrifugation of liver homogenate, approximately $90 \%$ of tetrahydrobiopterin is contained in the supernatant fraction.
\end{abstract}

Der Säugerorganismus scheidet verhältnismäßig viel Biopterin im Harn aus. Bilanzversuche an Ratten ergaben einen Wert von $40-60 \gamma$ pro Tag, der sich auch bei Fütterung der Tiere mit einer biopterinfreien Diät nicht änderte ${ }^{1}$. Versuche mit markiertem Biopterin zeigen seine große Stoffwechselstabilität sowohl in der Ratte ${ }^{2}$, als auch in der Honigbiene ${ }^{3}$ und in der Taufliege ${ }^{2,4}$. Das isotopenmarkierte Biopterin wird bei Versuchen in vivo zum großen Teil unverändert wiedergefunden. Andererseits wurden in neuerer Zeit eine ganze Reihe mischfunktioneller Oxygenasen beschrieben, die sich durch hydrierte Pterine aktivieren lassen ${ }^{5}$ und von denen im Falle der Phenylalanin-4-Hydroxylase (EC 1.14.3.1) Tetrahydrobiopterin als der natürliche Cofaktor identifiziert werden konnte ${ }^{6}$. Die vorliegende Untersuchungsreihe soll die Frage klären, in welcher Form das von der Ratte mit der Nahrung aufgenommene Biopterin im Organismus vorliegt.

\section{Material und Methodik}

Die Synthese von $\left(8 \mathrm{a}-{ }^{14} \mathrm{C}\right)$ Biopterin ${ }^{7}$ und von Tetrahydrobiopterin ${ }^{8}$ wurde an anderer Stelle beschrieben. Für die Inkubationsversuche haben wir die markierte

1 H. Kraut, W. Pabst, H. Rembold u. L. Wildemann, HoppeSeyler's Z. physiol. Chem. 332, 101 [1963].

2 H. Rembold, Pteridine Chemistry, Pergamon Press, Oxford 1964, S. 465.

3 H. Rembold u. G. Hanser, Hoppe-Seyler's Z. physiol. Chem. 319, 213 [1960].

4 H. Rembold, Angew. Chem. 73, 543 [1961].

5 S. Kaufman, Proc. nat. Acad. Sci. USA 50, 1085 [1963] ; T. Nagatsu, M. Levitt u. S. Udenfriend, J. biol. Chemistry
Verbindung mit einer spezifischen Aktivität von 0,15 bis $1,7 \mathrm{mC} / \mathrm{mMol}$ verwandt. Zur Bestimmung der Radioaktivität wurden Organstücke entweder in konzentrierter Ameisensäure gelöst oder ihr wasserlöslicher, mit 5-proz. Trichloressigsäure nicht fällbarer Anteil verwendet. Die Lösungen wurden auf Al-Plättchen aufgetragen und nach dem Trocknen im Omni-Guard (Tracerlab) bei einem Nulleffekt von unter 1 Imp/Min. vermessen.

Für die Versuche fanden Ratten der Stämme Wistar, Sprague Dawley und BAL im Alter von 6 Wochen und mit einem Gewicht von etwa $120 \mathrm{~g}$ Verwendung.

Aufarbeitung von Rattenlebern auf Pterine. Das Verfahren folgt weitgehend der bereits für die Isolierung von Pterinen aus Bienenpuppen beschriebenen $\mathrm{Me}$ thode ${ }^{9}$. Die Rattenlebern (250 g) wurden mit der gleichen Menge Eiswasser versetzt und im Mixer homogenisiert. Die bei niederer Tourenzahl sedimentierenden festen Bestandteile haben wir nochmals in der gleichen Weise zerkleinert und zentrifugiert. Aus den vereinigten Überständen fällt man durch allmähliche $\mathrm{Zu}$ gabe von 50-proz. Trichloressigsäure (TCE, Endkonzentration 5\%) unter starkem Rühren die löslichen Proteine und leichten Zellbestandteile aus. Die erhaltenen Niederschläge werden noch zweimal mit 3-proz. TCE extrahiert und der erhaltene Extrakt zusammen mit der Hauptmenge vorsichtig mit Äther extrahiert. Der letzte Rest von TCE läßt sich durch Perforieren mit Äther vollends entziehen. Der Leberextrakt wurde nach

239, 2910 [1964] ; A. R. Brennemann u. S. Kaufman, Biochem. biophysic. Res. Commun. 17, 177 [1964] ; S. NAKAmura, A. Ichiyama u. O. Hayaishi, Federat. Proc. 24, 604 [1965].

6 S. Kaufman, 1. c. ${ }^{5}$.

7 H. Rembold u. H. Metzagr, Chem. Ber. 96, 1395 [1963].

8 H. Rembold u. H. Metzger, Hoppe-Seyler's Z. physiol. Chem. 348, 194 [1967]. 
dem beschriebenen Standardverfahren ${ }^{9,10}$ auf seinen Pteringehalt untersucht. Der mit TCE fällbare Anteil wurde durch 5 -stdg. Kochen mit $500 \mathrm{ml} 5-n$. HCl hydrolysiert. Aus diesem Extrakt haben wir die Heterocyclen über mit 4\% Stearinsäure desaktivierte Aktivkohle ${ }^{11}$ abgetrennt und ebenfalls im Standardverfahren auf Dowex 1 X8 chromatographiert.

Bestimmung von Biopterin und Tetrahydrobiopterin durch Isotopenverdünnung. $15-50 \mathrm{~g}$ Leber bzw. die entsprechende Menge Zellpartikel werden in der 1,5-fachen Menge dest. Wasser 2 Min. lang in einem Homogenisator nach $\mathrm{Pot}$ er-Elveh jem zerkleinert. Dann tropft man unter starkem Rühren 30-90 $\gamma$ Biopterin $(1,7 \mathrm{mC} / \mathrm{mMol})$ in Wasser, bzw. die gleiche Menge Tetrahydrobiopterin in $0,1-n . \mathrm{HCl}^{8} \mathrm{zu}$. Die Lösung wird anschließend 20 Min. lang beschallt (MSE, $60 \mathrm{~W}$, Modell 3000), dann tropfenweise unter Rühren mit einer konz. TCE-Lösung versetzt bis zur Endkonzentration von $5 \%$ TCE und noch weitere 10 Min. lang gerührt. Den Niederschlag wäscht man zweimal mit 5-proz. TCE. Die vereinigten Extrakte, die bis zu $85 \%$ der eingesetzten Radioaktivität enthielten, extrahiert man zur Entfernung der TCE mit Äther und engt die dann neutrale Lösung im Rotationsverdampfer auf ein kleines Volumen ein. Biopterin wird daraus durch Chromatographie auf Ecteola-Cellulose und anschließende Feinreinigung auf Phosphor-Cellulose ${ }^{10}$ isoliert. Tetrahydrobiopterin zersetzt sich während der Aufarbeitung zu einem gewissen Prozentsatz zu Biopterin und wird mit diesem erfaßt. Die Biopterinmenge wird fluorometrisch durch Vergleich mit einer Eichkurve im Fluoreszenzzusatz zum Photometer Eppendorf bestimmt, seine Radioaktivität durch Messen eines Aliquots der Biopterinlösung im Omni-Guard.

\section{Ergebnisse}

\section{Biopterinaufnahme und Verteilung ${ }^{12}$}

Nach intraperitonealer Injektion von $14 \gamma\left(8 \mathrm{a}^{-14} \mathrm{C}\right)$ Biopterin $(1 \mathrm{mC} / \mathrm{mMol})$, gelöst in $1 \mathrm{~cm}^{3}$ physiologischer Kochsalzlösung, werden $84 \%$ der Radioaktivität in den ersten $24 \mathrm{Stdn}$., innerhalb eines weiteren Tages nochmals 6\% ausgeschieden. Die Gesamtausscheidung über die Niere beträgt in diesem Fall mindestens 90\%, obwohl die injizierte Substanzmenge weniger als der halben normalen Biopterinausscheidung pro 24 Stdn. entspricht. Völlig anders ist das Resorptionsverhalten nach Verfütterung der gleichen Substanzmenge mit einer Magensonde (Tab. 1). Biopterin wird fast vollkommen aufgenommen und tritt nur in geringer Menge wieder im Harn auf. Die Gesamtausscheidung der verabreich-

9 H. Rembold u. L. Buschmann, Liebigs Ann. Chem. 662, 72 [1963].

10 H. Rembold u. L. Buschmann, Hoppe-Seyler's Z. physiol. Chem. 330, 132 [1962].

\begin{tabular}{|c|c|c|c|c|}
\hline & \multicolumn{2}{|c|}{ Biopterin } & \multicolumn{2}{|c|}{ 7-Biopterin } \\
\hline & Kot & Harn & Kot & Harn \\
\hline \multirow{3}{*}{$\begin{array}{l}\text { am 1. Tag } \\
\text { am 2. Tag }\end{array}$} & 3,7 & 3,7 & 43 & 4,7 \\
\hline & 1,4 & 0,3 & 4 & 0 \\
\hline & 5,1 & 4,0 & 47 & 4,7 \\
\hline Summe & \multicolumn{2}{|c|}{9,1} & \multicolumn{2}{|c|}{51,7} \\
\hline
\end{tabular}

Tab. 1. Ausscheidung in \% der verabreichten Radioaktivität nach Verfüttern von $\left(8 \mathrm{a}^{-14} \mathrm{C}\right)$ Biopterin und -7-Biopterin an Ratten.

ten Radioaktivität liegt in diesem Fall bei 10 Prozent. Bemerkenswert ist ferner, daß die gleiche Menge des stellungsisomeren 7-Biopterins zum größten Teil nicht aufgenommen wird. In der Atemluft der Versuchstiere läßt sich keine radioaktive Kohlensäure nachweisen, die Substanz wird demnach nicht verbrannt.

Frühere mikrobiologische Analysen zeigten eine besonders hohe Crithidia-Aktivität in Leber, Nebenniere und Milz (Tab. 2). Zur Prüfung, ob sich eine

\begin{tabular}{llllll}
\hline Leber & 6,0 & Lunge & 0,9 & Serum & 0,2 \\
Nebenniere & 4,8 & Knochenmark 0,6 & Augen & 0,02 \\
Milz & 3,0 & Gehirn & 0,2 & & \\
Niere & 1,2 & Herz & 0,2 & & \\
\hline
\end{tabular}

Tab. 2. Verteilung der Crithidia-Aktivität auf Organe der Ratte, bezogen auf $\gamma$ Biopterin/g Organ ${ }^{2}$.

Parallele zwischen diesen Werten und dem Resorptionsverhalten im Falle des markierten Biopterins feststellen läßt, haben wir in mehreren Versuchen jeweils $14 \gamma$ markiertes Biopterin $\left(7 \cdot 10^{4} \mathrm{Imp} / \mathrm{Min}\right.$.) mit der Schlundsonde verfüttert und nach drei Tagen, d.h. am Ende einer meßbaren Ausscheidung, die Organe auf Radioaktivität untersucht (Tab.3).

\begin{tabular}{lcll}
\hline Serum & $220-330$ & Knochenmark & 0 \\
Leber & $340-540$ & Gehirn & 0 \\
Nebenniere & $50-70$ & Herz & 0 \\
Milz & 0 & & \\
Niere & 0 & Augen & 0 \\
Lunge & 0 & Thymus & 0 \\
\hline
\end{tabular}

Tab. 3. Radioaktivität in Imp/Min. in Organen der Ratte nach Verfütterung von $\left(8 \mathrm{a}-{ }^{14} \mathrm{C}\right)$ Biopterin $\left(7 \cdot 10^{4} \mathrm{Imp} / \mathrm{Min}.\right)$.

Sie lag nur bei zwei Organen und im Blutserum über der Nachweisgrenze. Auch stark proliferierende Zellen zeigen keinen höheren Einbau. Ratten, deren Lebern operativ auf die Hälfte des ursprünglichen Gewichts reduziert wurden, erhielten sechs oder 11 A. Asatoon u. C. E. Dalgliesh, J. chem. Soc. [London] 1956, 2291.

12 Teil der Dissertation H. Metzger, Univ München 1965. 
zwölf Stdn. nach erfolgter Operation $10 \gamma$ Biopterin $\left(8,4 \cdot 10^{4} \mathrm{Imp} / \mathrm{Min}.\right)$ verfüttert. Nach $12 \mathrm{Stdn}$. bzw. 3 Tagen fanden sich in der regenerierenden Leber 480 bzw. $510 \mathrm{Imp} /$ Minute. Auch mit Ascites beimpfte Ratten zeigen bei Untersuchung der in die Tumorzellen aufgenommenen Radioaktivität kein deutlicheres Bild als die teilhepatektomierten Tiere.

Trotz des geringen Einbaus wurde versucht, in der Leber vorhandene markierte Verbindungen zu isolieren, nachdem Vorversuche gezeigt hatten, daß Biopterin unter den Aufarbeitungsbedingungen weitgehend stabil ist. Bei der Chromatographie des mit TCE nicht fällbaren Leberextraktes wurde gleichzeitig nach Pterinen gesucht, welche als Intermediärprodukte einer Pterinbiosynthese diskutiert werden. Weder Neopterin, noch 6-Hydroxymethylpterin oder Pterin ließen sich nachweisen. Auch Isoxanthopterin und Violapterin, die in der Honigbiene vorkommen ${ }^{9}$, wurden nicht gefunden. Die Hauptmenge der Radioaktivität erschien am Anfang der Standardchromatographie auf Dowex $: X$ ô bei Elution mit Wasser in einem Bereich, wo stärker basische Stoffe als Biopterin zu erwarten sind. Die mit Wasser eluierbare Radioaktivität betrug insgesamt $73 \%$ der im Extrakt vorhandenen Aktivität, wovon $10 \%$ der Gesamtaktivität auf Biopterin entfielen, das in einer Menge von $250 \gamma$ isoliert werden konnte. Beim Versuch, die stärker basische radioaktive Substanzzone weiter zu reinigen, zersetzte sich das offensichtlich sehr labile Pterin. Im sauren Anteil des Leber- extraktes fanden sich geringe Mengen Pterincarbonsäure-(6), aber kein Biopterinphosphat. Im Hydrolysat des Leberproteins, dessen an Aktivkohle adsorbierbare Bestandteile nach dem gleichen Verfahren analysiert wurden, lassen sich keine Pterine nachweisen.

\section{Bestimmung von Biopterin und Tetrahydro- biopterin in der Rattenleber}

Die Frage, ob das im Leberextrakt enthaltene Biopterin auch in vivo in oxydierter, oder aber in reduzierter bzw. im Gemisch beider Formen vorliegt, läßt sich mit Hilfe der Isotopenverdünnungsmethode entscheiden. Tetrahydrobiopterin ist allerdings chemisch zu wenig stabil, um direkt bestimmt werden zu können. Bei seiner Rückoxydation entsteht aber immer in geringer Menge Biopterin, dessen spezifische Radioaktivität dann eine Berechnung des ursprünglich vorhandenen Tetrahydrobiopterins zuläßt. Zur Bestimmung der Isotopenverdünnung wurden die Lebern entweder unter Zusatz von $\left(8 \mathrm{a}-{ }^{14} \mathrm{C}\right)$ Biopterin oder von $\left(8 \mathrm{a}^{-{ }^{14}} \mathrm{C}\right)$ Tetrahydrobiopterin aufgearbeitet. Das Ergebnis dieser Untersuchungsreihe ist in Tab. 4 zusammengestellt.

Um einen Hinweis auf den möglichen Wirkungsort des Tetrahydrobiopterins zu bekommen, haben wir aus Rattenlebern auf dem üblichen Weg ${ }^{13}$ Zellfraktionen gewonnen und diese ebenfalls zusammen mit Tetrahydrobiopterin aufgearbeitet. Zum Ergebnis vgl. Tab. 5 .

\begin{tabular}{|c|c|c|c|c|}
\hline \multirow{3}{*}{$\begin{array}{c}\text { spez. Aktivität } \\
\text { in \% der einge- } \\
\text { setzten Aktivi- } \\
\text { tät }\end{array}$} & \multicolumn{4}{|c|}{ berechnete Gesamtmenge in $\gamma$} \\
\hline & pro g & ber & pr & \\
\hline & $\mathrm{BH}_{4}$ & B & $\mathrm{BH}_{4}$ & B \\
\hline 9,8 & 8,1 & - & 76 & - \\
\hline 85,5 & - & 0,40 & - & 3,5 \\
\hline 74,5 & - & 0,60 & - & 10,4 \\
\hline
\end{tabular}

Tab. 4. Bestimmung der Konzentration von Biopterin (B) und Tetrahydrobiopterin $\left(\mathrm{BH}_{4}\right)$ in Rattenlebern durch Zusatz der markierten Verbindungen.

\begin{tabular}{|c|c|c|c|c|c|c|c|c|}
\hline \multirow[t]{2}{*}{ Zellfraktion } & \multicolumn{2}{|c|}{ Rattenlebern } & \multirow{2}{*}{$\begin{array}{c}\text { eingesetzte } \\
\text { radioaktive } \\
\text { Substanz } \\
\text { in } \gamma / g \text { Leber }\end{array}$} & \multicolumn{2}{|c|}{$\begin{array}{c}\text { isoliertes Biopterin } \\
\text { abzüglich Träger, in } \gamma\end{array}$} & \multirow{2}{*}{$\begin{array}{l}\text { spez. Aktivität } \\
\text { in \% der ein- } \\
\text { gesetzten } \\
\text { Aktivität }\end{array}$} & \multicolumn{2}{|c|}{$\begin{array}{l}\text { berechnete Gesamt- } \\
\text { menge in } \gamma\end{array}$} \\
\hline & {$[\mathrm{n}]$} & {$[\mathrm{g}]$} & & pro g Leber & pro Leber & & $\begin{array}{l}\text { pro g } \\
\text { Leber }\end{array}$ & pro Leber \\
\hline Kerne & 2 & 12,6 & 3,24 & 0,067 & 0,42 & 94,5 & 0,55 & 3,5 \\
\hline Mitochondrien & 2 & 12,6 & 3,24 & 0 & 0 & 100 & 0 & 0 \\
\hline & 10 & 129 & 0,28 & 0,012 & 0,16 & 85 & 0,05 & 0,6 \\
\hline Mikrosomen & 4 & 51,5 & 0,52 & 0,012 & 0,16 & 70,5 & 0,21 & 2,8 \\
\hline Ưberstand & 2 & 12,6 & 3,24 & 0,500 & 3,15 & 28,4 & 8,1 & 51,5 \\
\hline
\end{tabular}

Tab. 5. Bestimmung der Konzentration von Tetrahydrobiopterin $\left(\mathrm{BH}_{4}\right)$ in Zellfraktionen von Rattenlebern durch Isotopenverdünnung.

13 W. C. Schneider u. G. H. Hogeboom, J. biol. Chemistry 183, 123 [1950]. 


\section{Diskussion}

Das Ausscheidungsverhalten für Biopterin ist bei der Ratte völlig verschieden, je nachdem die Sub. $\operatorname{stanz}$ injiziert oder über den Magen-Darm-Trakt aufgenommen wird. Offensichtlich wird Biopterin schon bei der Resorption aktiviert und in die Tetrahydroform umgewandelt, welche dann vom Körper in großer Menge gespeichert werden kann. Die sehr rasche und fast quantitative Ausscheidung des injizierten Biopterins über die Niere zeigt, daß dieses Organ die Verbindung nicht rückresorbiert und auf diese Weise die Biopterinkonzentration im Blut kontrolliert. In der Tat ist der Biopterinspiegel im Serum sehr niedrig. Deshalb könnte der Darm eine Biopterinreduktase enthalten. Im Blut kreisendes Biopterin wird nicht mehr in wesentlicher Menge reduziert. So wird auch der Fütterungsversuch mit 7-Biopterin verständlich. Dieses unnatürliche stellungsisomere Biopterin wird nicht aktiviert und wird deshalb nach Verfütterung im Kot ausgeschieden. Das resorbierte markierte Biopterin wird ziemlich gleichmäßig in die Körperzellen aufgenommen. Nur so ist zu verstehen, warum selbst in den biopterinreichsten Organen sich nur eine geringe Radioaktivität findet, die auch in stark proliferierenden Geweben nicht erhöht ist.

Der Versuch, aus der Leber radioaktive Metaboliten zu isolieren, führte gleichzeitig zu einer Bestätigung der Ergebnisse aus den Bilanzversuchen. Tetrahydrobiopterin wird sehr leicht zu teilhydrierten Verbindungen und nur in geringer Menge zu Biopterin oxydiert ${ }^{8}$. Dementsprechend entfallen nur $10 \%$ der Gesamtaktivität auf das voll oxydierte Produkt, während sich die Hauptaktivität auf sehr labile, schwach basische Verbindungen verteilt. Unsere bisherigen Ergebnisse sprechen für eine de novo Synthese des Biopterins in der Ratte ${ }^{1,14}$. Wir haben deshalb bei der Fraktionierung des Leberextraktes gleichzeitig geprüft, ob sich dort Neopterin, 6-Hydroxymethylpterin oder Pterin als mögliche Biopterinvorstufen finden lassen. Der Extrakt enthält nur Biopterin in einer Menge, die der mit dem mikrobiologischen Crithidia-Test bestimmten ent-

14 W. Pabst u. H. Rembold, Hoppe-Seyler's Z. physiol. Chem. 344, 107 [1966]. spricht. Natürlich ist damit nicht ausgeschlossen, daß labile Intermediärprodukte bei der Aufarbeitung verloren gingen. Biopterin ist kein Baustein für hochmolekulare Leberbestandteile. Nach Verfütterung von markiertem Biopterin wird keine Radioaktivität im mit Trichloressigsäure fällbaren Anteil gefunden. Auch nach saurer Hydrolyse unter Bedingungen, die Biopterin nicht zerstören, läßt sich diese Substanz nicht nachweisen.

Nach den Ergebnissen der Verdünnungsanalyse liegt Biopterin in der Rattenleber praktisch vollkommen in der hydrierten Form vor (Tab. 4). Das vor der Aufarbeitung zugesetzte markierte Tetrahydrobiopterin wird zusammen mit der bereits im Organ vorhandenen Verbindung in einer Ausbeute von rund $10 \%$ zu Biopterin zurückoxydiert und entsprechend verdünnt. Dieser Wert stimmt sehr gut mit den Daten überein, die wir bei der Auftrennung von radioaktiven Metaboliten aus Lebern nach Verfütterung von markiertem Biopterin erhalten haben. Aus der Verdünnung berechnet sich eine ursprüngliche Tetrahydrobiopterin-Menge von etwa $75 \gamma$ pro Leber. Ein Vergleich mit der entsprechenden Analyse nach Zusatz von markiertem Biopterin zeigt, daß in der Rattenleber höchstens 5\% der Tetrahydrobiopterin-Menge als Biopterin vorliegen können. Das hydrierte Biopterin findet sich bei der angewendeten Fraktionierungsmethode fast ausschließlich in der löslichen Zellfraktion (Tab. 5). Die in den Zellorganellen gefundenen Werte fallen demgegenüber sehr stark ab, doch läßt sich das Vorkommen einer geringen Menge Tetrahydrobiopterin nicht ausschließen. Wegen der günstigen Lage seines polarographischen Halbstufenpotentials ${ }^{8}$ haben wir in der Mitochondrienfraktion besonders sorgfältig nach hydriertem Biopterin gesucht. Wir konnten es allerdings nur in verschwindend geringer Konzentration nachweisen, und es wird noch weiterer Untersuchungen bedürfen, um die Frage einer Beteiligung von Tetrahydrobiopterin beim Wasserstofftransport in Mitochondrien zu klären.

Herrn Professor A. Butenande sind wir für die Förderung dieser Arbeit dankbar. Fräulein G. GALLINGER leistete wertvolle technische Hilfe. Die Untersuchungen wurden durch eine Sachbeihilfe der D e u t s c h e n Forschungsgemeinschaft wesentlich unterstützt. 